\title{
Management of complications in penile prosthesis reimplantation: A case report
}

\author{
Camilla Capretti $^{1}$, Antonio Avolio ${ }^{2}$, Alberto Florio ${ }^{2}$, Silvia Giovannozzi ${ }^{2}$, Domenico De Carolis ${ }^{2}$ \\ ${ }^{1}$ Department of Urology, "Ospedali Riuniti" University Hospital and Marche Polythecnic University, Ancona, Italy; \\ 2 Department of Urology, Mazzoni Hospital, Ascoli Piceno, Italy.
}

\begin{abstract}
Summary A 74-years-old patient, without comorbidity, underwent malleable penile prosthesis (MPP) implantation in 2007. In 2015, after a perineal trauma, he experienced stress urinary incontinence, medial extrusion of the left prosthetic cylinder and an urethrocavernous fistula. The cylinder was removed and an artificial urinary sphincter (AUS) implanted, together with a three-component inflatable penile prosthesis (IPP). The left corpus cavernosum (CC) was significantly shorter than the right one due to fibrosis.

After 8 months, partial lateral extrusion of the right prosthetic cylinder prompted a replacement with a shorter extensor. Six months after, a new diastasis of the ruptured area occurred due to a further CC shortening. The extensor was removed and the cylinder shortened, with a dermal graft applied to the area. Long-term patient satisfaction was high.
\end{abstract}

KEY WORDS: Erectile dysfunction; Penile prosthesis complications; Reimplantation.

Submitted 2 March 2020; Accepted 15 March 2020

\section{INTRODUCTION}

Managing the complications of penile prosthesis reimplantation is one of the biggest challenges in urology. Patients can develop corpus cavernosum (CC) scarring, with potential complications and penile shortening. In this study we present a patient with malleable penile prosthesis (MMP) who, after a perineal trauma, underwent inflatable penile prosthesis (IPP) implantation.

\section{Case report}

We present the case of a 74-years-old patient with no comorbidities, who underwent radical prostatectomy in 2005. Due to an erectile dysfunction not responding to less invasive methods, in 2007 a MPP (Subrini) was implanted. In 2015, due to an accident, the patient had a perineal trauma with symphysis pubis diastasis, resulting in stress urinary incontinence and medial extrusion of the left prosthetic cylinder at the level of the navicular fossa. This was due to the thinning and erosion of the tunica albuginea and the creation of an urethrocavernous fistula. Thus, the decision was taken to remove the cylinder and wait 6 months for a second surgery, to allow for scar tissue formation. The urethrography before the second surgery revealed a complete spontaneous healing of the fistula (Figure 1).

Consequently, the patient underwent the implantation of an artificial urinary sphincter (AUS) (Advance) and a three-component IPP (AMS 700-CX). During surgery, performed with penoscrotal approach, it occurred that the left CC was significantly shorter $(18 \mathrm{~cm})$ than the right one $(22 \mathrm{~cm})$, due to scarring and fibrosis.

The cylinders were implanted, a pump positioned in the scrotum and a $65 \mathrm{ml}$ reservoir inserted in the retro-pubic space. The prosthesis was activated intraoperatively. After 8 months, due to its partial lateral extrusion, the right prosthetic cylinder (which was $18+4 \mathrm{~cm}$ long) was extracted. The CC was now $19 \mathrm{~cm}$ long, compared to the previous $22 \mathrm{~cm}$. The $4 \mathrm{~cm}$-long extensor was substituted with a $1 \mathrm{~cm}$-long one and the cylinder replaced.

The prosthesis was activated intraoperatively, without pressure on the sutured area.

After 6 months, a new diastasis of the previous rupture area occurred, with lateral extrusion of the cylinder due to a further CC shortening by $2 \mathrm{~cm}$ (17 $\mathrm{cm}$ compared to the previous $19 \mathrm{~cm}$ ). The extensor $1 \mathrm{~cm}$ was removed and the cylinder shortened by $1.5 \mathrm{~cm}$ and re-positioned, with a total length gain of minus $2.5 \mathrm{~cm}$. Since the tunica albuginea was inadequate to cover parts of the cylinder, a graft of bovine dermal collagen (XENFORM) was applied with semi-continuous 2-0 absorbable monofilament (Vicryl) sutures to reinforce the area (Figure 2), restoring tunica albuginea continuity.

The patient had an uneventful postoperative course with no complications.

In 2019, a long-term, telephone-based follow-up showed a IIEF-5 score of 25 and no need for disposable incontinence products.

\section{Discussion}

In this case report, an intense fibrotic reaction occurred after removing the MPP right cylinder, leading to the shortening of the CC. When a MPP is removed and an IPP implanted, a further CC shortening occurs after surgery. While a MPP would always be active, the IPP may not be sufficiently activated by the patient, thus prompting further CC tissue rearrangement.

Therefore, it is advisable to encourage patients to inflate their prosthesis daily.

Implementing downsized prosthesis cylinders could also be a possible solution. If patients are uncomfortable with penile shortening, the conversion to standard cylinders (1), is usually possible within 8-12 months. According 


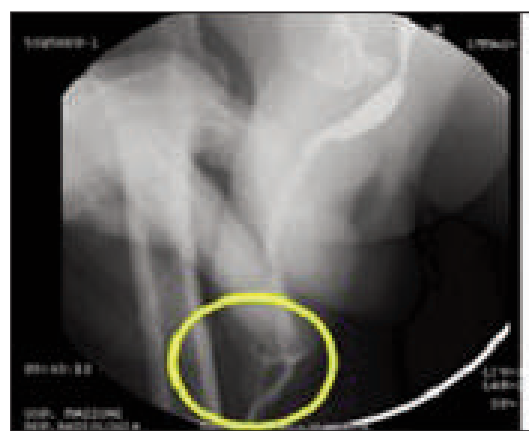

A.

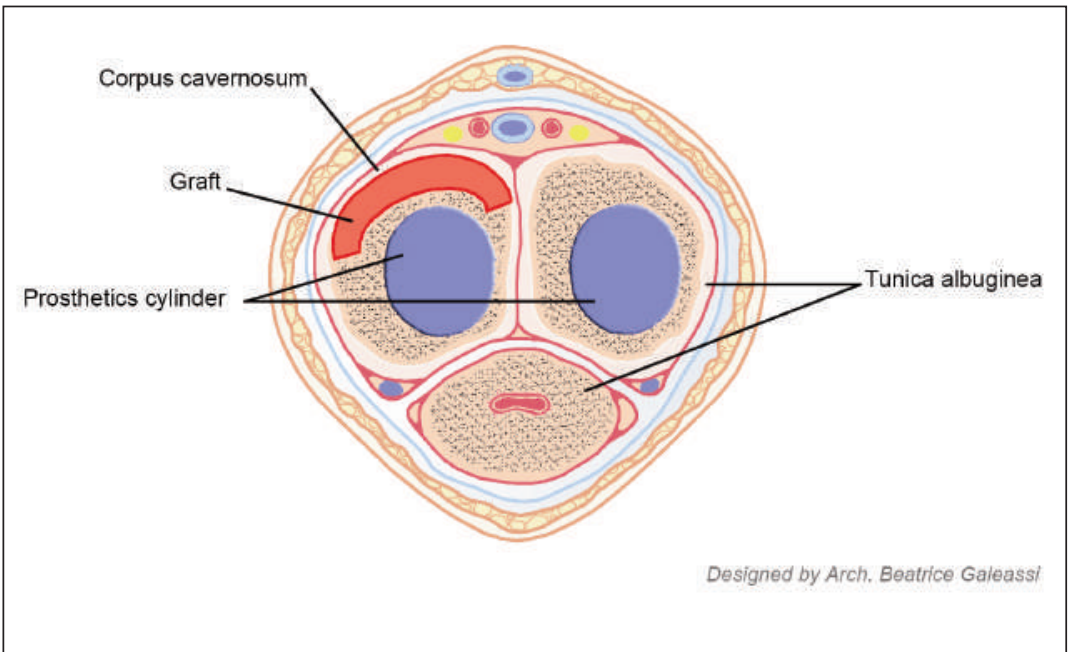

Figure 1.

A. Urethrography performed after the perineal trauma shows the urethrocavernous fistula (yellow circle); $B$. Urethrography repeated before the surgery shows the complete healing of the fistula (green square).

Figure 2.

Schematic representation of the graft of bovine dermal collagen (in red) applied to reinforce the tunica albuginea (in blu are represented the prosthetics cylinders). to literature, the combination of AUS and PPI does not adversely affect perioperative complications or device survival in relation to the device placement (2).

The use of grafts proved to be a safe option, resulting in no infections, rejections or discomfort for the patient. Moreover, long-term patient satisfaction proved to be high. In literature, there is no statistically significant difference in patient satisfaction between those undergoing PPI for the first time and those undergoing reimplantation (3).

\section{Conclusions}

Patients should always be informed of reimplantation risks and complications and encouraged to undergo surgery. The degree of CC fibrosis should always be taken in consideration. Graft implementation might be necessary, especially when transitioning from a MPP to an IPP. However, long-term patient satisfaction remains high.

\section{References}

1. Wilson SK. Reimplantation of inflatable penile prosthesis into scarred corporeal bodies. Int J Impot Res. 2003; 15(Suppl 5):S125-8.

2. Boysen WR, Cohen AJ, Kuchta K, et al. Combined placement of artificial urinary sphincter and inflatable penile prosthesis does not increase risk of perioperative complications or impact long-term device survival. Urology. 2019; 124:264-270. Available at: https://doi.org/10.1016/j.urology.2018.10.033.
3. Lledó-García E, Jara-Rascón J, Moncada Iribarren I, et al. Penile prosthesis first and replacement surgeries: analysis of patient and partner satisfaction. J Sex Med. 2015; 12:1646-1653. Available at: http://dx.doi.org/10.1111/jsm.12932.

\section{Correspondence}

Camilla Capretti, MD camilla.capretti2@gmail.com

Department of Urology, "Ospedali Riuniti" University Hospital and Marche Polythecnic University, Ancona, (Italy)

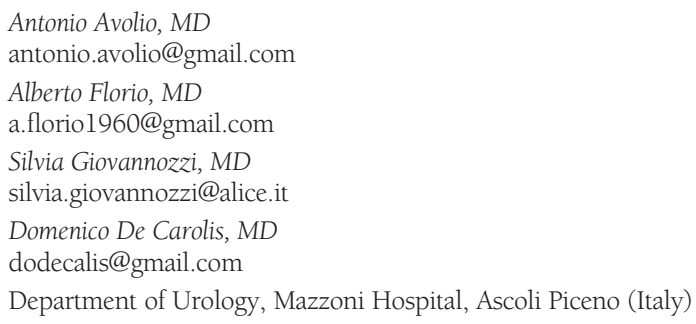

